\title{
Promoción de la Educación del Carácter mediante el CAS del Bachillerato Internacional
}

Enviado: 8 de octubre de 2021 / Aceptado: 18 de octubre de 2021 / Publicado: 31 de diciembre de 2021

ÁlVARO BALAGUER

Departamento Aprendizaje y Curriculum, Universidad de Navarra, España abalaguer@unav.es

(D) $0000-0002-8727-469$

MÓNICA SEPÚLVEDA

Facultad de Educación y Psicología, Universidad de Navarra, España

msepulvedas@unav.es

CONCEPCIÓN NAVAL

Departamento Teoría y Métodos de Investigación Educativa y Psicológica, Universidad de Navarra, España cnaval@unav.es

(D) 0000-0002-5927-9398

JUAN PABLO DABDOUB

Departamento Teoría y Métodos de Investigación Educativa y Psicológica, Universidad de Navarra, España jdabdoub@unav.es

(iD) $0000-0003-3750-0685$

DOI 10.24310/IJNE.8.2021.13664

\section{RESUMEN}

Este artículo analiza el componente CAS (Creatividad, Actividad y Servicio) del Bachillerato Internacional, y su relación con la Educación del Carácter. El CAS promueve fortalezas del carácter planteadas en distintos programas de Educación del Carácter. No obstante, en la bibliografía existente no se han identificado de manera explícita estas relaciones entre CAS y carácter. Con el objetivo de hallar los tipos de Educación del Carácter que se trabajan en el CAS, este trabajo presenta una revisión sistemática de la literatura (SLR) siguiendo el método PRISMA. Se encontraron 99 trabajos, de los cuales finalmente se in-

\section{ABSTRACT}

Promotion of Character Education through the CAS of the International Baccalaureate

This article analyzes the CAS component (Creativity, Activity and Service) of the International Baccalaureate, and its relationship with Character Education. The CAS promotes character strengths raised in different Character Education programs. However, these relationships between CAS and character have not been explicitly identified in the existing literature. In order to find the types of Character Education that are worked on in the CAS, this work presents a sys- 
cluyeron 17 en el análisis, tras aplicar los criterios de inclusión y exclusión. Distintas tipologías de Educación del Carácter aparecen reflejadas en todos ellos. La Educación del Carácter se encuentra inmersa en el programa CAS de diversos modos, tales como Educación en habilidades para la vida, Aprendizaje-servicio o Educación moral, entre otros. Por tanto, existe una dificultad en la literatura para definir carácter y Educación del Carácter, dado que es un concepto elástico y controvertido o, como algunos autores apuntan, un concepto paraguas.

Palabras Clave: Educación Internacional; Educación Ciudadana; Desarrollo Moral; Educación Religiosa. tematic review of the literature (SLR) following the PRISMA method. 99 works were found, of which 17 were finally included in the analysis, after applying the inclusion and exclusion criteria. Different typologies of Character Education are reflected in all of them. Character Education is immersed in the CAS program in various ways, such as Life Skills Education, Service-Learning or Moral Education, among others. Therefore, there is a difficulty in the literature to define character and Character Education, since it is an elastic and controversial concept or, as some authors point out, an umbrella concept.

Keywords: International Education; Citizenship Education; Moral Development; Religious Education.

\section{INTRODUCCIÓN}

La Organización de Bachillerato Internacional (IBO), es una entidad que pretende crear un mundo mejor y más pacífico a través de la educación. Para lograr este objetivo la IBO trabaja con colegios, universidades, gobiernos y otras organizaciones internacionales (IBO, 2019). A través de programas curriculares, la organización busca llevar sus principios fundacionales a la acción, desarrollando un perfil en la comunidad de aprendizaje que intenta "formar personas con mentalidad internacional que, conscientes de la condición que las une como seres humanos y de la responsabilidad que comparten de velar por el planeta, contribuyan a crear un mundo mejor y más pacífico” (IBO, 2019).

El primer programa curricular con el que comenzó la IBO hace más de 50 años, es el Programa del Diploma del Bachillerato Internacional. Este programa fue creado para ofrecer a los alumnos, entre los 16 y 19 años, una educación integral que facilita la movilidad geográfica y cultural y fomenta el entendimiento entre personas de distintos países. Para ello se estructuró un modelo que contiene seis áreas disciplinares y tres componentes nucleares que complementan el desarrollo de habilidades de pensamiento, de investigación, personales y sociales. Uno de estos componentes es CAS (creatividad, actividad y servicio) que posibilita, a través de diferentes experiencias, alcanzar objetivos de aprendizaje en los alumnos relacionados con su autoconocimiento, su relación con los otros, su compromiso con el entorno, la evaluación y reflexión sobre sus actos, entre otros (IBO, 2015). 
El componente nuclear CAS del Programa del Diploma nació tras un par de años de funcionamiento del modelo académico, con el objetivo de que la formación que recibieran los alumnos no solo fuese académica o disciplinar, sino que formara a la persona de manera holística. Por lo tanto, se crea CAS como una oportunidad de educar más allá de lo académico, buscando desarrollar la parte creativa y estética, física y de servicio social (IBO, 2015). El añadir la parte de servicio social nace del profesor Kurt Hahn, del Colegio Internacional Atlantic College, que tenía como convicción que los aspectos físico y social son claves para desarrollar el carácter (Van Oord, 2010).

A través del CAS se busca que los alumnos desarrollen la conciencia de sí mismos y el sentido de identidad, la importancia de sus decisiones y el impacto que pueden tener a nivel local o global. Mediante los diferentes proyectos que los alumnos desarrollan se busca su crecimiento personal y social. Es un elemento del modelo del Programa del Diploma que les ayuda a adquirir el compromiso y la perseverancia para alcanzar las metas y los objetivos que se proponen (IBO, 2015).

\subsection{La promoción del carácter a través del IB}

Esta formación de la persona en su totalidad que pretende la IBO a través de su modelo y, en concreto, a través del CAS, sugiere la búsqueda de la formación del carácter a través de la educación. Una Educación del Carácter (EC) entendida como ofrecer medios a las personas para que actúen libremente, dando relevancia a las virtudes características de un ser humano pleno (Naval, 2000).

La IBO, al ser una organización internacional, que se encuentra en más de 150 países alrededor del mundo (IBO, 2019), sugiere que cada colegio desarrolle en sus alumnos un perfil de aprendizaje que cuenta con diez atributos que se relacionan directamente con el carácter. Los diez atributos son: ser indagadores, conocedores, pensadores, comunicadores, de principios, de mente abierta, afectuosos, tomadores de riesgos, equilibrados y reflexivos. Estos atributos sitúan la EC junto con la educación académica como una característica que define a un colegio IB (Bailey \& Cooker, 2018).

El perfil de la comunidad de aprendizaje busca que, a lo largo de la escolaridad, los alumnos sean conscientes de que la formación va más allá del éxito académico y pretende que los profesores, los directivos y los familiares fomenten estas capacidades y responsabilidades humanas. Se evidencia entonces que la IBO se propone la formación de seres humanos conscientes de su libertad y de la importancia de aprender a respetarse a sí mismos, a los demás y al mundo que 
los rodea (IBO, 2019). De hecho, el perfil de aprendizaje del Bachillerato Internacional (International Baccalaureate, IB) se basa en cierto modo en un enfoque de EC (Van Oord, 2013).

Se puede entender entonces que el componente CAS, dentro del programa del Diploma, promueve de alguna manera la EC, a través de sus objetivos de aprendizaje y el desarrollo del perfil de aprendizaje de la comunidad IB. Sin embargo, existe una escasez de literatura científica que aborde de manera explícita esta promoción de la EC por parte del perfil de aprendizaje del IB. Aunque se ha considerado este objetivo para el Primary Years Programme del IB (Mattix Foster \& Daly, 2016) o de manera global para el IB (Bailey \& Cooker, 2018), falta literatura científica que examine estas relaciones para programas que oferte el IB destinados a estudiantes adolescentes.

Partiendo de estas carencias en la literatura previa, consideramos que resulta especialmente interesante a nivel científico y aplicado investigar estas relaciones entre el CAS y la EC. Por un lado, en torno al tipo específico de EC que promueve el programa CAS. Para ello, planteamos una revisión sistemática de la literatura que analice ambas cuestiones.

\subsection{Objetivos}

El objetivo principal de esta investigación es llevar a cabo una revisión sistemática de las investigaciones publicadas acerca del CAS (Creatividad, Actividad, Servicio) que muestren una relación con elementos de la educación del carácter. De manera concreta, perseguimos encontrar las evidencias en torno a las relaciones entre el CAS y distintos tipos de educación del carácter que se trabajan en el programa.

\section{MÉTODO}

Se ha llevado a cabo una revisión sistemática a través del formato PRISMA (Statement of Transparent Reporting of Systematic Reviews and Meta-Analyses) (Moher et al., 2009) para presentar revisiones sistemáticas con o sin metaanálisis (Urrútia \& Bonfill, 2010), cuyas directrices se aplicaron para la búsqueda y selección de los documentos.

En primer lugar, se acordó la estrategia de búsqueda entre los autores del trabajo para, posteriormente, extraer los datos teniendo como referencia los artículos que abordan el programa CAS en su relación con la EC. 


\subsection{Preguntas de investigación}

Para llevar a cabo los objetivos mencionados anteriormente, se han planteado dos preguntas de investigación que contribuyen a recoger la información necesaria para analizar las evaluaciones. La pregunta de investigación a la que atendemos es la siguiente: ¿Cuáles son los elementos de la educación del carácter que se han relacionado con el CAS?

\subsection{Estrategia de búsqueda y criterios de inclusión}

Se ha realizado la búsqueda bibliográfica de documentos indexados en dos bases de datos: Scopus y Web of Science. Ambas son internacionales y tienden a ser las más usadas para búsquedas científicas en todos los campos. Además, también se recopilaron aquellos documentos de la revisión sistemática anual que realiza el propio IB, que respondían a nuestras cuestiones de investigación.

Se recogieron aquellos documentos en los cuales en el título, resumen y palabras clave contasen cualesquiera de las combinaciones de las palabras clave siguientes: IB and CAS or creativ* $^{*}$ or activit* or service. La búsqueda se ha restringido a publicaciones comprendidas entre enero de 2010 y febrero de 2020, escritas en el idioma inglés o español, y documentos del tipo artículo, revisión, capítulo de libro/libro y editorial material. La problemática estudiada adquiere relevancia desde hace diez años. De hecho, la propia IBO lleva a cabo revisiones sistemáticas desde 2012. Buscamos trabajos que incluyesen los elementos del área del CAS como parte del Programa del Diploma del IB. La tabla 1 muestra los criterios de inclusión y exclusión.

Tabla 1. Criterios de inclusión y de ex clusión

\begin{tabular}{lll}
\hline Publicación & Criterios de inclusión & Criterios de exclusión \\
\hline 1. Aborda el IB de maner a explícita & Sí & No \\
\hline 2. Preguntas de inv estigación & Responde & No responde \\
\hline 3. Tipo & Informe, artículo, libro o capítulo. & $\begin{array}{l}\text { Proceeding paper, conference paper, disserta- } \\
\text { tion, thesis, final grade, master }\end{array}$ \\
\hline 4. Idiomas & Inglés, español & Idiomas distint os a inglés o esp añol \\
\hline
\end{tabular}

Se reunieron 99 títulos con sus respectivos abstracts. La figura 1 muestra un diagrama de filtrado PRISMA con los procesos de inclusión, la descripción de los estudios descartados y la razón de su eliminación. 
Figura 1. Diagrama de flujo asado en PRISMA p ara la selec ción de estudios incluidos en el met aanálisis

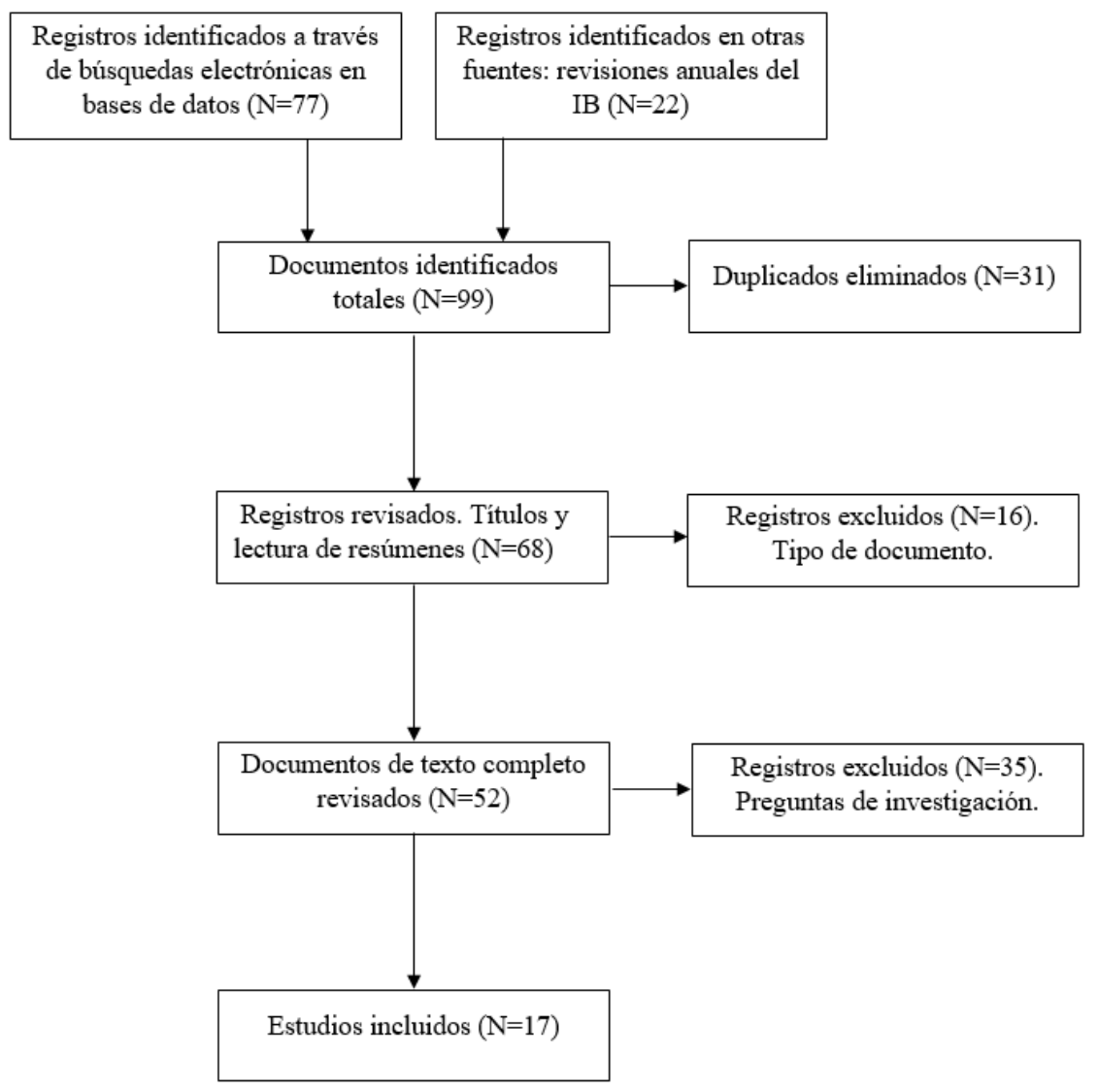

\subsection{Procedimiento}

La selección de documentos se llevó a cabo en dos fases. Primeramente, se procedió a la eliminación de los documentos duplicados. Seguidamente, se eliminaron aquellos que no formaban parte del tipo de documento planteado. Finalmente, se desecharon los que no guardaban relación con el IB o con las preguntas de investigación planteadas.

Los documentos finalmente seleccionados (Tabla 2) fueron categorizados atendiendo a dos características. En primer lugar, abordar alguno o varios de los componentes del CAS. En segundo lugar, guardar relación con la EC. A través del análisis de cada texto la información que respondía a las preguntas de investigación se fue extrayendo. De este modo, se determinó la asociación entre éstas, y así se alcanzaron las conclusiones de la investigación. La información recogida de cada documento fue la siguiente: año de publicación, base de datos en la que consta, país(es) en el/los que se recabó la muestra del estudio, tamaño de la muestra, tipo de documento, tipo de investigación, tipo de diseño de investigación, tipo de EC, resultados del carácter y detalles métricos (https://url2.cl/IutVk). 
Tabla 2. Publicaciones incluidas en la r evisión sist emática

1 Lillo, S. R. (2019). In pursuit of community engagement: Unpacking the knowledge and skills associated with servicelearning efforts. Journal of Research in International Education, 18(1), 3-22. https://doi.or g/10.1177/1475240919829997

2 Dickson, A., Perry, L. B., \& Ledger, S. (2018). Impacts of International Baccalaur eate programmes on teaching and learning: A review of the literature. Journal of Research in International Education, 17(3), 240-261. https://doi.or g/10.1177/1475240918815801

3 Hayden, M., \& Mclntosh, S. (2018). International education: the transformative potential of experiential learning. Oxford Review of Education, 44(4), 403-413. https://doi.or g/10.1080/03054985.2017.1402757

4 Martin, R. A., Tanyu, M., \& Perry, S. (2016). Structures and programme supports for creativity, action, service in the international baccalaur eate diploma programme: An implementation study in Turkey. Journal of Research in International Education, 15(2), 120-136. https://doi.or g/10.1177/1475240916659722

5 Hatziconstantis, C., \& Kolympari, T. (2016). Student perceptions of Academic Service Learning: Using mixed content analysis to examine the effecti eness of the International Baccalaur eate Creativity, Action, Service programme. Journal of Research in International Education, 15(3), 181-195. https://doi.or g/10.1177/1475240916668074

6 Wasner, V. (2016). Critical service learning: A participatory pedagogical approach to global citizenship and international mindedness. Journal of Research in International Education, 15(3), 238-252. https://doi.or g/10.1177/1475240916669026

7 Wright, E., \& Lee, M. (2014). Elite International Baccalaur eate Diploma Programme schools and inter-cultural underst anding in China. British Journal of Educational Studies, 62(2), 149-169. https://doi.or g/10.1080/00071005.2014.924615

8 Wright, E., \& Lee, M. (2014). Developing skills for youth in the 21st century: The role of elite International Baccalaur eate Diploma Programme schools in China. International Review of Education, 60(2), 199-216. https://doi.or g/10.1007/s11159-0149404-6

9 Jamison, C. (2013). 'God has created me to do him some defini e service' (Cardinal Newman): vocation at the heart of the Catholic curriculum. International Studies in Catholic Education, 5(1), 10-22. https://doi.or g/10.1080/19422539.2012.754593

10 DiGiorgio, C. (2010). Choices of students, parents, and teachers and their effects on schools and communities: A case study of a new enriched high school program. Journal of School Choice, 4(3), 278-292. https://doi.or g/10.1080/15582159.20 10.504107

11 Billig, S. H., \& Good, B. M. (2013). International Baccalaureate Diploma Programme: Study of North and South American students' civic-mindedness. RMC Rese arch Corpor ation.

12 Billig, S. H. (2017). Service and Service-Learning in IB High Schools. International Journal of Research on Service-Learning and Community Engagement, 5(1).

13 Hayden, M., Hemmens, A., McIntosh, S., Sandoval-Hernández, A., \& Thompson, J. (2017). The impact of creativity, action, service (CAS) on students and communities. International Bac calaur eate Organization

14 Brodie, T. (2014). The perception and practice of Creativity, Action, Service in the International Baccalaureate Diploma Programme for students, teachers and schools. http://www.ibo.org/contentassets/4ccc99665bc04f3686957ee197c13855/finale ecutivesummaryandappendices.pdf

15 Cannings, J., Piaggio, M. I., Brodie, T. \& Muir, P. (2015). Creativity, Activity, Service (CAS) for the IB Diploma: an essential guide for students. Cambridge Univ ersity Press

16 Palmer, N. (2018). Emergent constellations: Global citizenship education and outrospective fluenc . Journal of Research in International Education, 17(2), 134-147. https://journals.sagepub .com/doi/abs/10.1177/1475240918793963

17 Van Oor d, L. (2010). Kurt Hahn's mor al equivalent of w ar. Oxford Review of Education, 36(3), 253-265. 


\section{RESULTADOS}

\subsection{Variables extrínsecas al proceso científico}

De los 17 documentos incluidos, 13 de ellos son artículos, 3 de ellos informes, y 1 de ellos libro. Atendiendo a su procedencia, un 70,6 \% de las editoriales o revistas a las que corresponden los documentos incluidos son de Reino Unido y un 17,6 \% pertenecen a las publicaciones del IB, con sede en Suiza. El resto corresponden a EE.UU. y Holanda, con una publicación en cada país.

La figura 2 muestra la relación de las revistas o editoriales y la cantidad de documentos encontrados. La revista que ha publicado un mayor número de artículos que incluyen el CAS como uno de los temas principales a abordar es el Journal of Research in International Education (1, 2, 4, 5, 6 y 15), seguida del Oxford Review of Education (3, 17). A la par, la International Baccalaureate Organization (IBO) ha publicado tres informes con esta temática (11, 13 y 14).

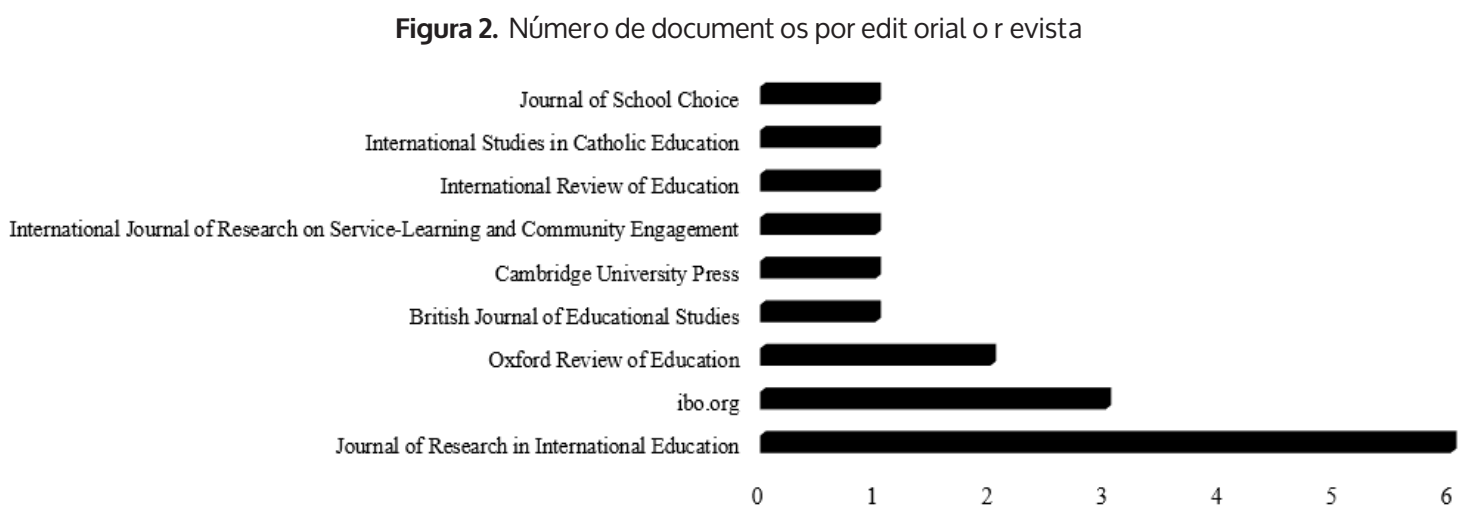

Por lo que respecta a la dimensión temporal de las publicaciones incluidas, el último artículo hallado en nuestra investigación es el item 1 "In pursuit of community engagement: Unpacking the knowledge and skills associated with service-learning efforts" (Lillo, 2019). Las publicaciones en torno al CAS han ido oscilando, pero manteniéndose una tendencia lineal a nivel evolutivo.

Figura 3. Número de publicaciones por año

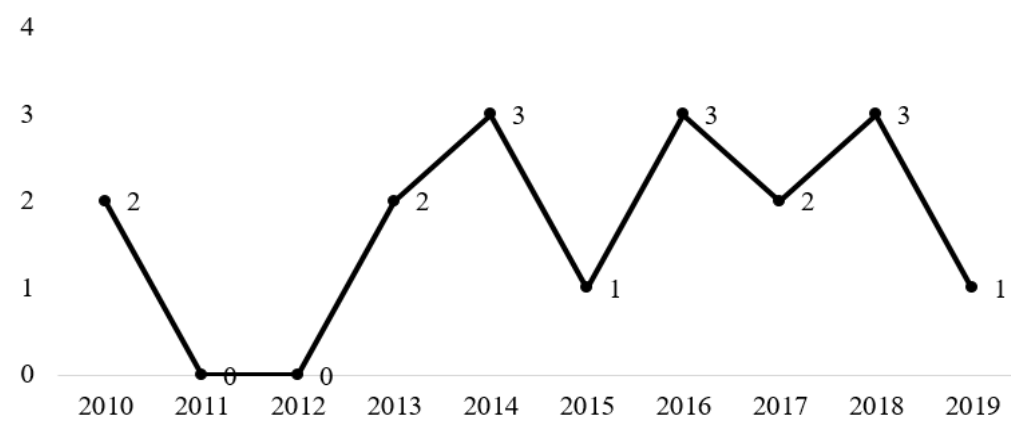


Atendiendo al sexo de los autores de los documentos incluidos se aprecia una similaridad entre ellos. De los 27 autores, 13 de ellos (el 48,1\%) son hombres y 14 de ellas son mujeres (el $51,9 \%)$. Del total de autores, $16(59,3 \%)$ se encuentran vinculados a instituciones universitarias, mientras que $11(40,7 \%)$ ejercen su labor profesional en instituciones no universitarias (Fig. 4). Cinco de los autores de estas publicaciones pertenecen a la University of Bath, como institución con un mayor número de ellos. Le sigue la Murdoch University, con 3 autores. La Ibicus-International Baccalaureate (IB) Training es la institución no universitaria más representada, seguida de la RMC Research Corporation, con 3 y 2 autores, respectivamente.

Figura 4. Distribución por centual de publicaciones por tipo de institución asociada a aut or(es)

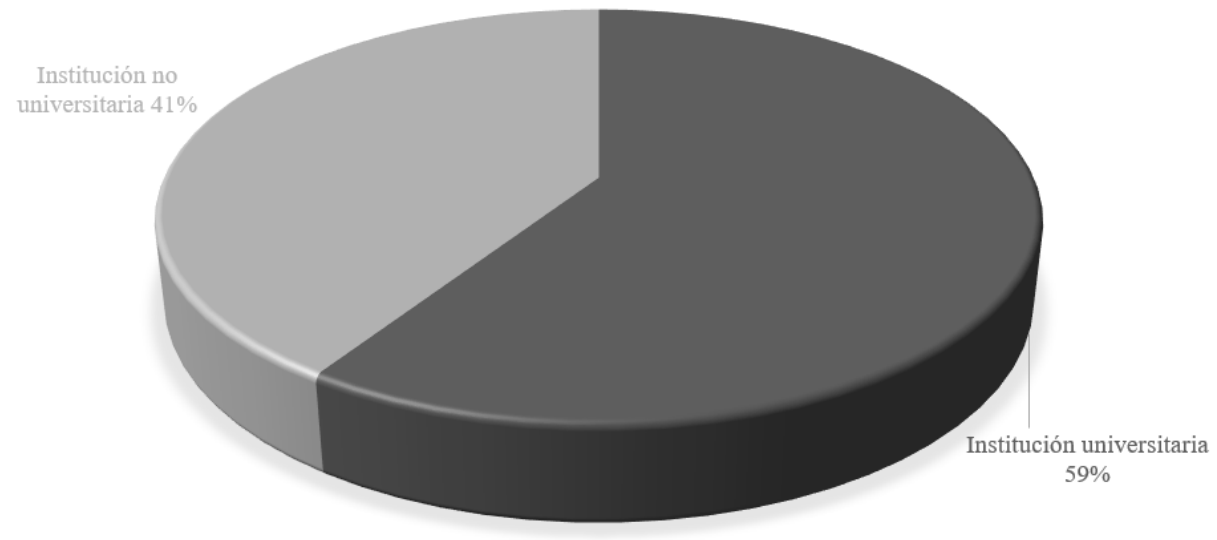

\subsection{Variables metodológicas}

En lo concerniente a las variables metodológicas y en función del tipo de investigación de las publicaciones, como ya se ha mencionado, encontramos una guía aplicada para seleccionar actividades CAS (15). El resto de documentos pueden dividirse entre cinco de tipo teórico (2, 3, 6,9 , y 17) y once trabajos empíricos $(1,4,5,7,8,10,11,12,13,14$ y 16). Las publicaciones teóricas revisan la literatura científica mientras que los trabajos empíricos utilizan una muestra ad hoc. En la figura 5 aparecen representados los porcentajes de distribución.

La revista Oxford Review of Education tiende a publicar artículos teóricos, mientras que la página oficial de International Baccalaureate (ibo.org), publica resultados empíricos. La revista Journal of Research in International Education, con mayor número de artículos en esta revisión, publica investigaciones de distinto tipo, ya que busca cerrar la brecha entre la teoría emergente y la práctica diversa en todo el mundo, considerando las implicaciones educativas que tiene una educación internacional (Journal of Research in International Education, 2020). 
Figura 5. Distribución por centual de publicaciones por tipo de inv estigación

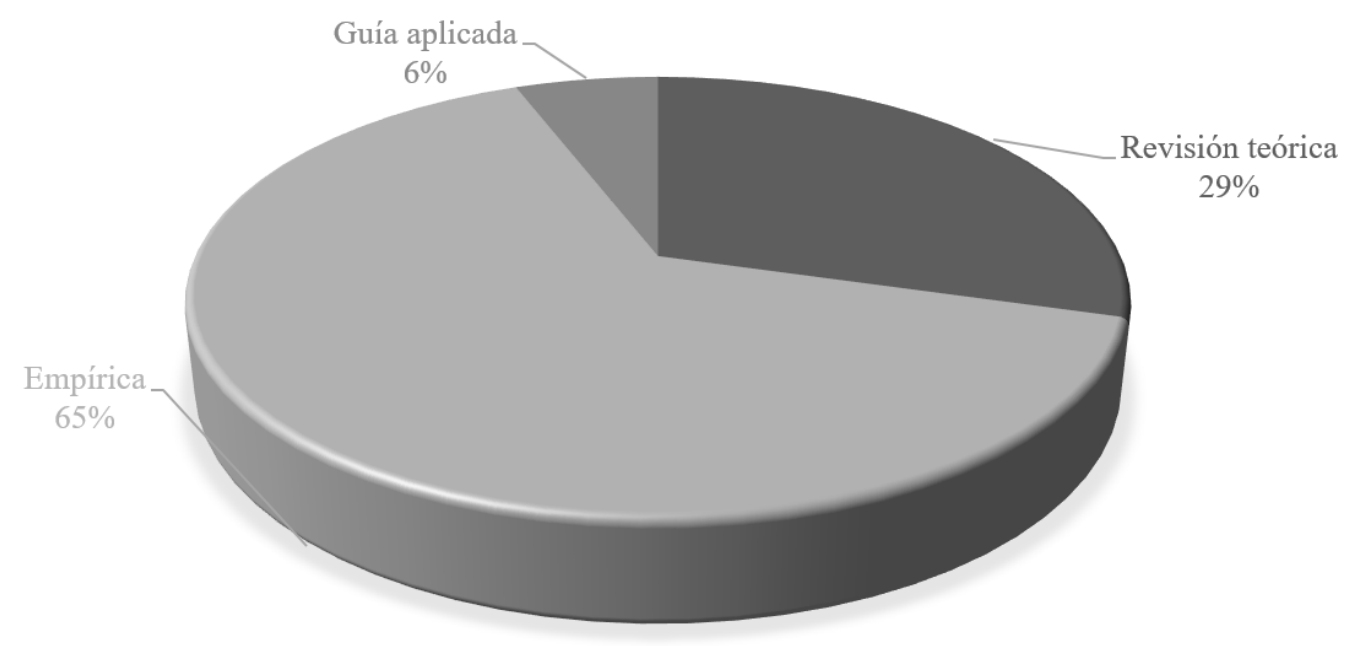

La tabla 3 muestra la información estadística de la procedencia geográfica de los colegios de las muestras por continentes. La mayor parte de los estudiantes de los artículos empíricos proceden de colegios situados en países asiáticos. No obstante, en proporción a la población total de los continentes, el africano es el que contaría con menor representación $(1,13)$. Si atendemos a la representación de la muestra por país en la que se sitúan los colegios, la figura 6 muestra una mejor representación de los países europeos. De manera ampliada puede consultarse en el siguiente enlace: https://url2.cl/8hanj

Tabla 3. Procedencia ge ográfica de los colegios de las muest as por continent es

\begin{tabular}{llll}
\hline Región & Muestra & & \\
\hline & N & $\%$ & Proporción poblacional \\
\hline Europe & 3002 & 32,3 & $4,04 \mathrm{e}-4$ \\
\hline Asia & 4040 & 43,5 & $8,79 \mathrm{e}-5$ \\
\hline Africa & 325 & 3,5 & $2,46 \mathrm{e}-5$ \\
\hline America & 1411 & 15,2 & $1,45 \mathrm{e}-4$ \\
\hline Oceania & 502 & 5,4 & $1,22 \mathrm{e}-3$ \\
\hline TOTAL & $\mathbf{9 2 8 0}$ & $\mathbf{1 0 0}$ & $\mathbf{1 , 2 1 e - 4}$ \\
\hline
\end{tabular}

Nota. Uno de los artículos (14) no pr oporciona dat os sobr e estudiant es por escuela en cada p aís. Por lo tanto, los dat os para esa muestr a no se r eflejan en la abla. 
Figura 6. Distribución por centual de publicaciones empíricas por continent e

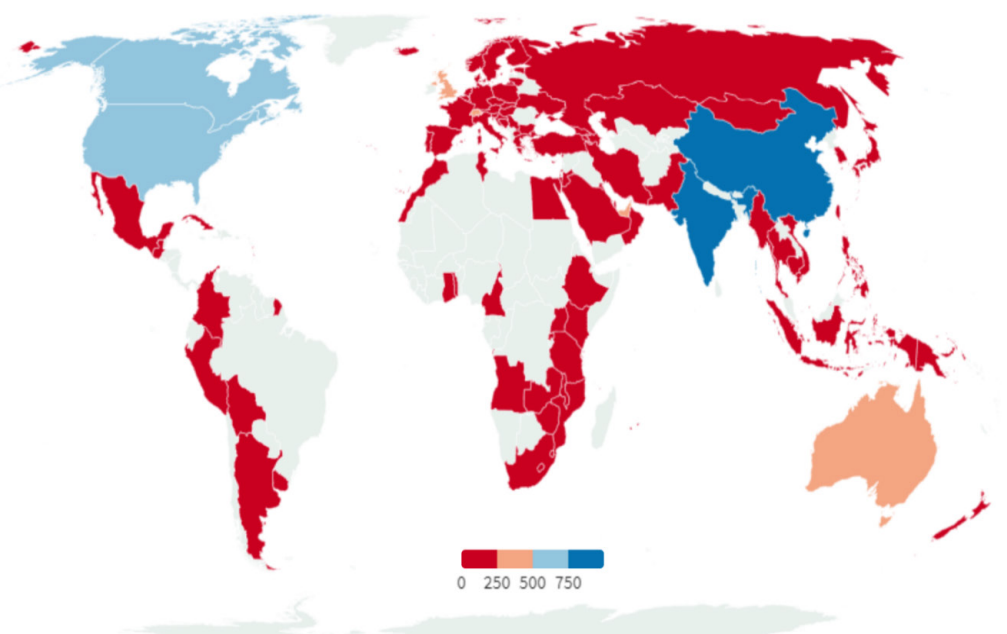

Atendiendo al tipo de diseño utilizado en los artículos empíricos, siete de ellos utilizan exclusivamente una metodología cualitativa (1, 4, 5, 7, 8, 10 y 16) mientras que solo un artículo emplea únicamente un diseño cuantitativo (12). Tres artículos plantean un diseño que combina técnicas cuantitativas y técnicas cualitativas. La entrevista semiestructurada constituye la técnica más utilizada en estas investigaciones, utilizada por todos los artículos con metodología cualitativa o mixta (11, 13 y 14). Cuatro artículos utilizan grupos focales $(4,11,13$ y 14) y cuatro artículos $(11,12,13$ y 14) emplean cuestionarios para recabar información, tanto los de metodología mixta como cuantitativa en particular.

El análisis de la información se realiza en la mayor parte de estudios utilizando métodos inductivos de análisis de datos (e.g., enfoque de la teoría fundamentada constructivista: 16). Sin embargo, también se combina con métodos deductivos en otros artículos. El programa de análisis más usado es Nvivo (4, 7 y 8). También hay algunos análisis con dos fases (11, 12 y 14), especialmente en artículos con metodología mixta.

Por lo que respecta al tamaño muestral, este suele ser mayor en aquellas investigaciones de naturaleza cuantitativa, buscando una mejor representatividad poblacional, así como una mayor generalización de los resultados. Obviamente, los artículos con diseño exclusivamente cualitativo han recabado un número muestral inferior, en especial aquellos que se limitan a entrevistas semiestructuradas (1, 5, 7, 8, 14 y 16). En la tabla 4 se muestra la media del tamaño de la muestra de los artículos empíricos, cuyos datos ponen de manifiesto una variación destacada del tamaño de la muestra en función del tipo de diseño. 
Tabla 4. Tamaño de muestr a promedio de artículos empíricos según el tipo de diseño

\begin{tabular}{lll}
\hline Tipo de diseño & \multicolumn{2}{l}{ Media tamaño muestral } \\
\hline & Estudiantes & Adultos* \\
\hline Cualitativo & 26 & 13,75 \\
\hline Cuantitativo & 3058,6 & 490 \\
\hline
\end{tabular}

Nota. Coordinador es CAS, profesores, dir ectores, etc.

En todos los artículos empíricos se recogen estudiantes del programa CAS en las muestras. Algunos trabajos incluyen sujetos no participantes en el programa CAS en los grupos focales o antiguos alumnos en las encuestas (11, 12 y 13). Tan solo un $18 \%$ de estos artículos recogen una muestra exclusivamente formada por estudiantes (5 y 12). Los adultos participantes varían de padres a profesores, pasando por directores, antiguos alumnos (alumni), coordinadores CAS, administrativos del colegio, consejo escolar y departamento provincial de educación.

\subsection{Variables de Educación del Carácter}

En lo que respecta a la temática incluida en EC, se han clasificado los tipos de EC que han abordado los distintos artículos en seis categorías: Educación moral (5, 11, 12, 14 y 17), Educación religiosa (9 y 17), Educación en Habilidades para la vida (1, 2, 3, 4, 5, 6, 7, 8, 10, 11, 13, 14, 15, 16 y 17), Aprendizaje-servicio (1, 2, 4, 5, 6, 7, 8, 9, 10, 11, 12, 13, 14, 15 y 17), Educación cívica y ciudadanía $(1,2,4,5,6,7,10,11,12,15$ y 16) y Mentalidad global (1, 2, 3, 6, 7, 10, 11, $12,13,14,15$ y 16). La figura 7 representa los porcentajes de las temáticas de la EC abordadas por los documentos analizados.

Figura 7. Distribución por centual de publicaciones por tipo de Educación del Car ácter abor dado

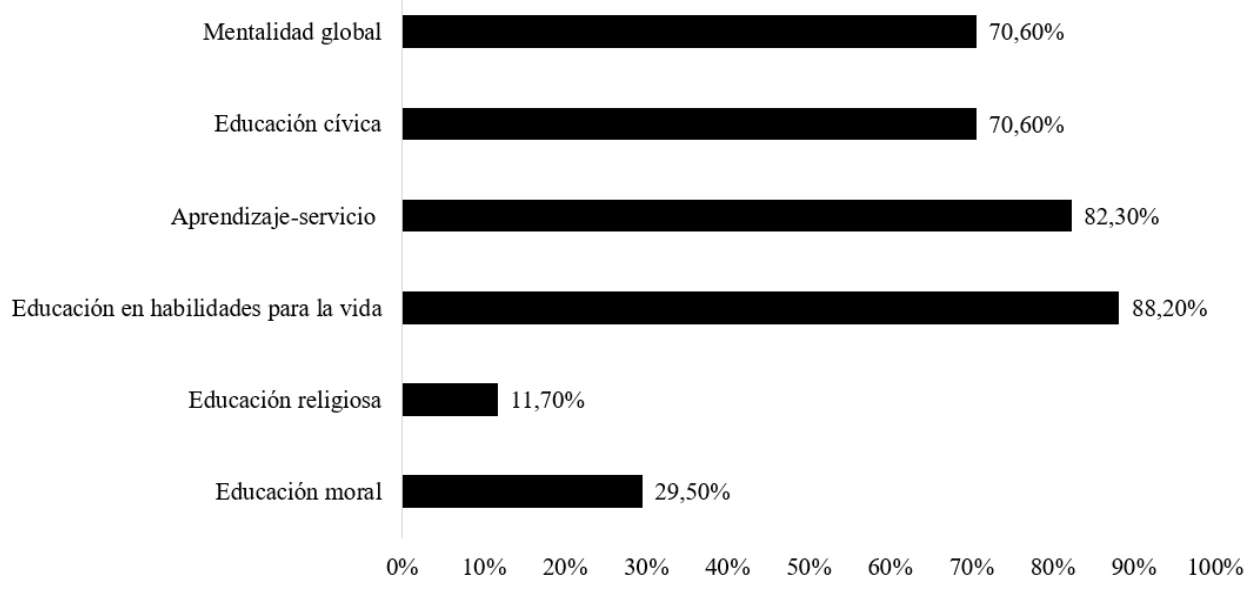


En el ámbito de la Educación moral se incluyen temas como la educación o el razonamiento moral o el desarrollo ético. Cinco de los documentos analizados analizaban enfoques humanitarios en los estudiantes, la ética de servicio, la implicación ética o moral de un filósofo particular (5, 11, 12, 14 y 17). La Educación religiosa en relación con el CAS ha sido abordada en dos artículos teóricos (9 y 17) (e.g. catholic ethos, God's purpose in life). Aquí la EC se entiende en un contexto de tradición de fe, justificando la moralidad desde una fuente trascendente.

Algunas de las Habilidades para la vida (Life Skills) han sido tratadas en la gran mayoría de los documentos, entendidas como habilidades de tipo socioemocional. Es decir, habilidades prácticas o comunicativas y otras actitudes sociales positivas, como la iniciativa o motivación (4), el desarrollo socioemocional (4), la responsabilidad (5, 11 y 17), la resolución de problemas interpersonales (5), el entendimiento social (1, 5 y 7) o algunas competencias relacionadas con la autorregulación (2 y 6). Los más comunes han sido la comunicación (1, 2, 5, 8, 10 y 13), el liderazgo $(1,2,4,5,8$ y 11) o las relaciones con los demás (1, 13 y 15).

Además, el Aprendizaje-Servicio - entendido como experiencias "prácticas" de servicio comunitario integradas en el curriculo- ha sido abordado en un total de 14 publicaciones de manera implícita, haciendo referencia de manera más frecuente a community service $(10,15$ y 17), pero también a términos como service projects (4), service activities (7, 8, 11 y 13), service work (7), service experiences (13) o service others (9). Sin embargo, tan solo en seis se ha mencionado Service Learning de manera explícita (1, 5, 6, 12, 14 y 15), o bien mediante otras derivaciones como Critical Service Learning (6), Academic Service Learning (5) o Service Learning Pedagogy (1).

La Educación cívica pretende la preparación para la futura ciudadanía, así como la transmisión de valores cívicos. Este tipo de educación clave en el programa CAS se ha considerado relevante en once documentos $(1,2,4,5,6,7,10,11,12,15$ y 16), todos ellos convergiendo en cómo se ha fortalecido la conducta cívica en los estudiantes. Términos como civic development (4), civic participation (5), civic action (5), civic engagement (15), civic skills (11) o civic attitudes (11) han sido los más utilizados al mencionar este tipo de EC.

Otro de los términos más recurrentes en las publicaciones ha sido el término global citizenship. Precisamente, la educación para la Mentalidad global ha sido investigada en doce documentos $(1,2,3,6,7,10,11,12,13,14,15$ y 16). Además de la "ciudadanía global", se ha aludido con frecuencia a términos como, global issues (1, 3 y 13) o education for internationalism (17) para designar a esta categoría, en este orden de frecuencia. 


\section{DISCUSIÓN}

Esta investigación ha pretendido llevar a cabo una revisión sistemática de las publicaciones del CAS en su relación con la EC. Aunque la organización IB lleve publicando desde hace 8 años una revisión anual en torno al CAS, nuestra motivación respondía a una ausencia de revisiones que mostraran esta relación con la EC. De hecho, el CAS ofrece un panorama alentador para la promoción de la EC, a través de un amplio abanico de posibilidades en cuanto a recursos y actividades en ámbitos personales y sociales.

En los últimos años se ha generado un aumento de las publicaciones que investiguen los resultados positivos del CAS. Tomando en consideración tanto las características de las revistas que contienen los artículos, como la organización a la que pertenecen los informes y la editorial del libro, se ha apreciado una ausencia de publicaciones en revistas o editoriales españolas. Por el contrario, la gran mayoría de las editoriales o revistas que han publicado estos trabajos han sido británicas. Cabe tener en cuenta que Reino Unido es el tercer país del mundo con mayor número de colegios IB, tras EE.UU. y Canadá (IBO, 2020). Pese al aumento de publicaciones en los últimos años en torno al CAS el presente proceso de revisión sistemática ha dado como resultado 17 documentos de las bases de datos Wos y Scopus, junto con los documentos de las revisiones anuales de la propia IBO (excluyendo trabajos universitarios o en proceso de publicación).

La revista fournal of Research in International Education ha resultado la que ha publicado un mayor número de artículos que relacionen el CAS con distintos resultados en torno al Carácter o a la EC, con seis publicaciones. Esta revista cuenta con el doble de publicaciones que la propia organización ibo.org en torno a esta temática concreta. La Oxford Review of Education cuenta con dos artículos.

Se ha encontrado una práctica igualdad en cuanto al género de los autores de los documentos analizados. Semejante proporción también se ha comprobado en lo que respecta a la filiación de los autores entre instituciones universitarias y no universitarias, lo cual pone de manifiesto la predominancia del contexto británico en los contextos de publicación. Tanto en investigadores como en editoriales o revistas en la investigación en CAS. De hecho, la Universidad de Bath ha trabajado desde los inicios del IB como centro de referencia en investigación e innovación, además de asesorar a la IBO.

Atendiendo a las variables metodológicas, la distribución de las muestras por colegios pone de relieve la prevalencia de países europeos. Por el contrario, el continente americano 
y el africano cuentan con menor representación, en especial este último. Ello da cuenta de la distancia que todavía separa a este continente del resto en desarrollo socioeconómico (Darham \& Araujo, 2016). Un tanto sustancial es que el continente africano cuenta con un menor número de colegios de IB. En este sentido, el contexto cultural en que se desarrolla el CAS puede marcar una diferencia significativa en las posibilidades didácticas. De hecho, la falta de desarrollo influye negativamente en los esfuerzos de los facilitadores (e.g. líderes estudiantiles, profesores, coordinadores, etc.) (Lillo, 2019).

Por lo que respecta a las variables relacionadas con la EC implicadas en el programa CAS, se han encontrado seis tipos distintos de EC: Mentalidad global, Educación cívica, Aprendizajeservicio, Educación en habilidades para la vida, Educación religiosa y Educación moral. Cabe resaltar la dificultad de clasificar la EC, puesto que ésta representa un paraguas (Arthur, 2003, in Arthur, 2017; Kristjánsson, 2015: 20, 45; Williams, 2000) que abarca tipos a la hora de clasificarla para elaborar programas orientados a promover resultados. En efecto, la EC, la educación moral y la educación para la ciudadanía tienen literatura superpuesta pero diferenciada (Althof y Berkowitz, 2006). De todos modos, la adquisición de alguna habilidad en la mayoría de las publicaciones revela el claro sentido promotor que adquiere el CAS para los estudiantes.

Debido al componente de servicio del CAS no es de extrañar que el Aprendizaje-Servicio haya sido objeto de análisis frecuente en estas publicaciones, así como la educación cívica y para la ciudadanía. Un amplio porcentaje de las publicaciones analizadas ponen de manifiesto la intención del IB de formar un tipo de ciudadano con un marcado perfil global. Resnik (2008) afirmó que las cualidades y disposiciones fomentadas en el perfil de aprendizaje del IB coinciden con las del ciudadano ideal y del trabajador global ideal. La promoción de una mentalidad global ha sido abordada por la inmensa mayoría de las publicaciones analizadas, entrelazándose con esa educación cívica. En efecto, tal como sostiene Lillo (2019: 4):

Existe poco consenso en la literatura sobre las definiciones de ciudadanía global, participación comunitaria y aprendizaje de servicio [...]. Los conceptos entrelazados de ciudadanía global, participación cívica, participación comunitaria, servicio y aprendizaje de servicio no son lo mismo. Sin embargo, las líneas borrosas entre estos conceptos cambian según el teórico o el participante.

En este sentido, se hace necesaria la colaboración, no solo de las familias y/o comunidades, sino también construir comunidad; comunidades locales que participen activamente. Nos referimos a vínculos comunitarios que proporcionen capital social en pro de la educación (Bolívar, 2020; Bolívar \& Luengo, 2020). 
El perfil de aprendizaje del IB está firmemente fundamentado en la EC y, particularmente en la educación moral (Van Oord, 2013). De hecho, la promoción de componentes éticos y morales por medio del programa se ha encontrado en prácticamente la mitad de los documentos analizados. Sin embargo, no se hace mención explícita al término "moral" o a la educación moral, salvo en publicaciones que, de manera conjunta, aborden la educación religiosa, puesto que el IB es una organización secular (Gardner-McTaggart, 2016), restringiendo el uso de términos religiosos a cuando el programa se aplica en alguna institución con esos principios.

En efecto, aunque la educación religiosa ha sido abordada en un 11,7\% de las publicaciones analizadas, el liderazgo escolar del IB, en general, adopta el pluralismo a través de la coherencia del cristianismo. De hecho, los valores cristianos se encuentran omnipresentes en la orientación de los directores del IB en el contexto europeo occidental (Gardner-McTaggart, 2019). Además, la Educación para la Ciudadanía Global en los planes de estudio del IB representa un componente religioso, frecuente en los sistemas escolares de élite, pero en su forma globalizada: secular e inclusiva, a la vez que equitativa y distinta (Gardner-McTaggart, 2016).

De todos modos, el estudio de estas cuestiones lleva a preguntarse cuál es el papel que puede desempeñar el IB en la educación de los estudiantes y en qué medida el CAS puede contribuir a la EC, más allá del aspecto teórico y técnico. Por lo tanto, las distintas experiencias que viven los estudiantes en el seno del programa CAS promueven y/o desarrollan la EC mediante el desarrollo de distintas capacidades y competencias en el alumnado. De hecho, la EC está en el corazón del sistema del IB (Bailey \& Cooker, 2018).

\subsection{Limitaciones}

A nivel de estudio y de resultado, una de las principales limitaciones que presenta esta revisión sistemática atiende a los documentos que puede abarcar la búsqueda bibliográfica. Efectivamente, pueden existir publicaciones que investiguen el impacto de alguno o varios de los componentes del CAS -Creatividad, Actividad y Servicio- en la EC. No obstante, no han sido hallados al no encontrarse estos términos $-\mathrm{u}$ otros términos relacionados- incluidos en las palabras clave, resumen y/o título del documento.

En este sentido, uno de los principales problemas al abordar las revisiones sistemáticas es encontrar todas las investigaciones relevantes. Hemos utilizado las principales bases de datos con una búsqueda automática. Sin embargo, analizando la revisión anual que proporciona IB, se han encontrado otros documentos que no aparecían en tales bases de datos. A ello cabe añadir que solo se ha llevado a cabo tal búsqueda en las dos bases de datos que consideramos más apropiadas a este efecto. 
Por otro lado, a nivel de revisión, nuestra investigación viene a resaltar que no pueden llevarse a cabo metaanálisis acerca de los documentos incluidos en nuestro análisis de manera que puedan producir resultados que cuenten con validez y fiabilidad. En efecto, la mayoría de los documentos son, bien trabajos teóricos, bien investigaciones cualitativas. Y, en menor medida, estudios que utilicen muestras amplias con diseños cuantitativos. Además, algunos trabajos empíricos presentan una ausencia de datos, tales como el tamaño global de la muestra (e.g. Brodie, 2014; Martin et al., 2016) o la diferenciación del tamaño muestral por países (e.g. Brodie, 2014). Ya se ha puesto de manifiesto previamente la escasez de diseños de investigación en programas de IB que controlen los factores de confusión o permiten extraer inferencias causales, por lo que convendría que futuras investigaciones atiendan a los sesgos de selección (Dickson et al., 2018).

Finalmente, otra de las limitaciones apunta al planteamiento de las preguntas de investigación. Nos referimos a la dificultad en la conceptualización del carácter y de la EC, que es un concepto elástico y controvertido. Se ha hecho mención a la característica de "paraguas" del término carácter, pero cabe también apuntar a la diversidad de competencias, habilidades, capacidades o cualidades que se engloban bajo este término, con pensamientos, sentimientos y acciones que se consideran dimensiones del carácter (Lickona, 1996).

\subsection{Futuras investigaciones}

Se hacen necesarias investigaciones experimentales que utilicen procesos estadísticos con diseños potentes que incluyan controles y diseños longitudinales. Nos referimos a investigaciones que permitan comparar tamaños del efecto del CAS en distintos resultados relacionados con la EC.

De todos modos, resulta todavía escasa la cantidad de investigaciones que comparen estas relaciones, con independencia de la naturaleza del diseño. De hecho, los datos analizados permitieron encontrar en el segmento temporal investigado en las bases de datos Scopus y WOS, 99 artículos, si bien tan solo 17 han mostrado investigar de manera directa o indirecta la relación entre el CAS y la EC.

Además, sería especialmente útil que las futuras investigaciones en esta línea recogiesen muestras amplias y culturalmente diversas (e.g. Billig, 2017; Billig \& Good, 2013; Hayden et al., 2017). Si bien también es interesante que combinen ambos tipos de diseño (e.g. Brodie, 2014; Hayden et al., 2017) de manera que ofrezcan mayores posibilidades para la interpretación de 
los resultados. De este modo, podrían llevarse a cabo metaanálisis que arrojasen más evidencias de las propiedades del CAS para potenciar la EC.

\section{CONCLUSIONES}

La presente revisión constituye un estudio novedoso, debido a la ausencia de investigaciones que muestren relaciones explícitas entre el programa CAS del IB y la promoción de resultados del carácter en el marco de la EC. En este sentido, aporta distintos elementos de análisis sobre la evolución y la extensión de los elementos del CAS dentro del Programa de Diploma del IB y su impacto en los resultados positivos en los estudiantes. Resultados entendidos como carácter, dentro del amplio paraguas de la EC. Asimismo, también se ha analizado la promoción de la EC en sus distintos tipos de aplicación.

Además, de cara al ámbito aplicado, esta revisión pone de relieve la necesidad de que los centros educativos consideren su papel promotor de desarrollo personal y social, así como su importancia en la formación de los docentes (Torrijos et al., 2018). El hecho de que un perfil de aprendizaje pueda ayudar a transformar significativamente la vida de los estudiantes, supone un avance importante en unos tiempos caracterizados por el cambio y la internacionalización.

En este sentido, los retos y amenazas que afrontan las escuelas son numerosos. Para aquellas que tratan de no sucumbir a la lógica utilitarista, nuestros resultados pueden apoyar investigaciones posteriores, por un lado, de IB en general, y de Programa de Diploma y CAS en particular. Por otro lado, de la EC en general y, de sus distintos tipos en particular. La base de datos de los documentos analizados puede resultar un apoyo para investigaciones que se focalicen en otras dimensiones teóricas o prácticas. Asimismo, estas aportaciones pueden aportar orientaciones para los profesionales aplicados a la hora de llevar a cabo programaciones curriculares.

\section{REFERENCIAS BIBLIOGRÁFICAS}

Althof, W. \& Berkowitz, M. W. (2006) Moral education and character education: Their relationship and roles in citizenship education. Fournal of Moral Education, 35(4): 495-518. https://doi. org/10.1080/03057240601012204

Arthur, J. (2003). Education with Character: The moral economy of schooling. London: Routledge. 
Arthur, J. (2017). Convergence on policy goals: Character education in East Asia and England. fournal of International and Comparative Education (FICE), 5(2), 59-71. https://doi.org/10.14425/ jice.2016.5.2.59

Bailey, L., \& Cooker, L. (2018). Who Cares? Pro-social education within the programmes of the International Baccalaureate. Journal of Research in International Education, 17(3), 228-239. https:// doi.org/10.1177/1475240918816405

Billig, S. H. (2017). Service and Service-Learning in IB High Schools. International fournal of Research on Service-Learning and Community Engagement, 5(1).

Billig, S. H., \& Good, B. M. (2013). International Baccalaureate Diploma Programme: Study of North and South American students' civic-mindedness. RMC Research Corporation.

Bolívar, A. (2020). En la reapertura de las escuelas: Reconstruir la comunidad. Cuadernos de Pedagogía, 512, 112-116.

Bolívar, A., \& Luengo, F. (2020). El currículo global de la sostenibilidad desde el eje escuela-familiacomunidad (cap. 4). En Moya, J. y Valle, J.M. (Coords.): La reforma del currículo escolar: ideas y propuestas, 35-52. ANELE-REDE.

Brodie, T. (2014). The perception and practice of Creativity, Action, Service in the International Baccalaureate Diploma Programme for students, teachers and schools. http://www.ibo.org/contentassets/ 4ccc99665bc04f3686957ee197c13855/finalexecutivesummaryandappendices.pdf

Cannings, J., Piaggio, M. I., Brodie, T. \& Muir, P. (2015). Creativity, Activity, Service (CAS) for the IB Diploma: an essential guide for students. Cambridge University Press.

Darham, C. M., \& Araujo, L. (2016). Underdeveloped African Education: The root of its poverty. Sci Insigt Edu. https://doi.org/10.15354/sie.16.re008

Dickson, A., Perry, L. B., \& Ledger, S. (2018). Impacts of International Baccalaureate programmes on teaching and learning: A review of the literature. Fournal of Research in International Education, 17(3), 240-261. https://doi.org/10.1177/1475240918815801

DiGiorgio, C. (2010). Choices of students, parents, and teachers and their effects on schools and communities: A case study of a new enriched high school program. fournal of School Choice, 4(3), 278-292. https://doi.org/10.1080/15582159.2010.504107

Gardner-McTaggart, A. (2016). International elite, or global citizens? Equity, distinction and power: the International Baccalaureate and the rise of the South. Globalisation, Societies and Education, 14(1), 1-29. https://doi.org/10.1080/14767724.2014.959475 
Gardner-McTaggart, A. (2019). International schools' leadership and Christianity. Globalisation, Societies and Education, 17(4), 458-473. https://doi.org/10.1080/14767724.2018.1558047

Grupo de Investigación en Educación, Ciudadanía y Carácter (2020). https://www.unav.edu/web/facultad-de-educacion-y-psicologia/educacion-y-ciudadania

Hayden, M., Hemmens, A., McIntosh, S., Sandoval-Hernández, A., \& Thompson, J. (2017). The impact of creativity, action, service (CAS) on students and communities. International Baccalaureate Organization

Hayden, M., \& McIntosh, S. (2018). International education: the transformative potential of experiential learning. Oxford Review of Education, 44(4), 403-413. https://doi.org/10.1080/03054985.201 $\underline{7.1402757}$

Hatziconstantis, C., \& Kolympari, T. (2016). Student perceptions of Academic Service Learning: Using mixed content analysis to examine the effectiveness of the International Baccalaureate Creativity, Action, Service programme. Journal of Research in International Education, 15(3), 181-195. https://doi.org/10.1177/1475240916668074

IBO (2019). ¿Qué es la educación del IB?. IB Publishing, p 11.

IBO (2015). Programa del Diploma: Guía Creatividad, Actividad y Servicio. IB Publishing, p. 2-13.

IBO (2020). https://www.ibo.org

Jamison, C. (2013). 'God has created me to do him some definite service' (Cardinal Newman): vocation at the heart of the Catholic curriculum. International Studies in Catholic Education, 5(1), 10-22. https://doi.org/10.1080/19422539.2012.754593

Journal of Research in International Education (2020). Retrieved from https://journals.sagepub.com/ aims-scope/JRI

Kristjánsson, K. (2015). Aristotelian character education. Routledge. pp. 20, 45.

Lickona, T. (1996). Eleven principles of effective character education. fournal of Moral Education, 25(1), 93-100.

Lillo, S. R. (2019). In pursuit of community engagement: Unpacking the knowledge and skills associated with service-learning efforts. Journal of Research in International Education, 18(1), 3-22. https://doi.org/10.1177/1475240919829997

Martin, R. A., Tanyu, M., \& Perry, S. (2016). Structures and programme supports for creativity, action, service in the international baccalaureate diploma programme: An implementation study in Turkey. Fournal of Research in International Education, 15(2), 120-136. https://doi. org/10.1177/1475240916659722 
Mattix Foster, A. A., \& Daly, K. (2016). Focus on Elementary: Creating Global Citizens: Using Attitudes and Action to Teach Character Education: Patricia Crawford and April A. Mattix Foster, Editors. Childhood Education, 92(1), 80-85. https://doi.org/10.1080/00094056.2016.1134251

Moher, D., Liberati, A., Tetzlaff, J., Altman, D. G., \& The PRISMA Group. (2009). Preferred reporting items for systematic reviews and meta-analyses: The PRISMA statement. PLoS Med, 6(7), e1000097. https://doi.org/10.1371/journal.pmed1000097

Palmer, N. (2018). Emergent constellations: Global citizenship education and outrospective fluency. fournal of Research in International Education, 17(2), 134-147. https://journals.sagepub.com/doi/ abs/10.1177/1475240918793963

Resnik, J. (2008). The construction of the global worker through international education. In: J. Resnik (ed.). The production of educational knowledge in the global era. Sense Publishers.

Torrijos, P., Martín, J. F., \& Rodríguez, M. J. (2018). La educación emocional en la formación permanente del profesorado no universitario. Profesorado, Revista de Currículum y Formación del Profesorado, 22(1), 579-597.

Urrútia, G., \& Bonfill, X. (2010). Declaración PRISMA: una propuesta para mejorar la publicación de revisiones sistemáticas y metaanálisis. Medicina clínica, 135(11), 507-511. https://doi. org/10.1016/j.medcli.2010.01.015

Van Oord, L. (2010). Kurt Hahn's moral equivalent of war. Oxford Review of Education, 36(3), 253-265.

Van Oord, L. (2013). Moral education and the International Baccalaureate learner profile. Educational Studies, 39(2), 208-218. https://doi.org/10.1080/03055698.2012.717260

Wasner, V. (2016). Critical service learning: A participatory pedagogical approach to global citizenship and international mindedness. fournal of Research in International Education, 15(3), 238-252.

Williams, M. M. (2000). Models of character education: Perspectives and developmental issues. The Journal of Humanistic Counseling, Education and Development, 39(1), 32-40.

Wright, E., \& Lee, M. (2014). Developing skills for youth in the 21st century: The role of elite International Baccalaureate Diploma Programme schools in China. International Review of Education, 60(2), 199-216. https://doi.org/10.1007/s11159-014-9404-6

Wright, E., \& Lee, M. (2014). Elite International Baccalaureate Diploma Programme schools and intercultural understanding in China. British fournal of Educational Studies, 62(2), 149-169. https:// doi.org/10.1080/00071005.2014.924615 